\title{
Extensive deamidation at asparagine residue 279 accounts for weak immunoreactivity of tau with RD4 antibody in Alzheimer's disease brain
}

Ayaho Dan ${ }^{1,4}$, Muneaki Takahashi ${ }^{1}$, Masami Masuda-Suzukake ${ }^{1}$, Fuyuki Kametani ${ }^{1}$, Takashi Nonaka ${ }^{1}$, Hiromi Kondo ${ }^{2}$, Haruhiko Akiyama ${ }^{3}$, Takao Arai ${ }^{4}$, David MA Mann ${ }^{5}$, Yuko Saito ${ }^{6}$, Hiroyuki Hatsuta ${ }^{7}$, Shigeo Murayama ${ }^{7}$ and Masato Hasegawa ${ }^{1^{*}}$

\begin{abstract}
Background: Intracytoplasmic inclusions composed of filamentous tau proteins are defining characteristics of neurodegenerative tauopathies, but it remains unclear why different tau isoforms accumulate in different diseases and how they induce abnormal filamentous structures and pathologies. Two tau isoform-specific antibodies, RD3 and RD4, are widely used for immunohistochemical and biochemical studies of tau species in diseased brains.

Results: Here, we show that extensive irreversible post-translational deamidation takes place at asparagine residue 279 (N279) in the RD4 epitope of tau in Alzheimer's disease (AD), but not corticobasal degeneration (CBD) or progressive supranuclear palsy (PSP), and this modification abrogates the immunoreactivity to RD4. An antiserum raised against deamidated RD4 peptide specifically recognized 4R tau isoforms, regardless of deamidation, and strongly stained tau in AD brain. We also found that mutant tau with N279D substitution showed reduced ability to bind to microtubules and to promote microtubule assembly.

Conclusion: The biochemical and structural differences of tau in AD from that in $4 R$ tauopathies found in this study may therefore have implications for prion-like propagation of tau.
\end{abstract}

Keywords: Alzheimer's disease, Tau, Deamidation, Aging, Microtubule

\section{Background}

Intracellular inclusions composed of filamentous tau proteins are defining characteristics of many neurodegenerative diseases, including Alzheimer's disease (AD), Pick's disease, corticobasal degeneration (CBD), and progressive supranuclear palsy (PSP). Tau is a microtubule-associated protein that stabilizes microtubules and promotes their assembly. In adult human brain, 6 tau isoforms are expressed as a result of mRNA splicing. They are divided into two groups, 3-repeat (3R) and 4-repeat (4R) tau isoforms, according to whether or not exon 10 is expressed. Tau pathologies show clear morphological differences among different diseases or disease types, and different tau isoforms are accumulated in the diseased

\footnotetext{
* Correspondence: hasegawa-ms@igakuken.or.jp

'Department of Neuropathology and Cell Biology, Tokyo Metropolitan

Institute of Medical Science, Setagaya-ku, Tokyo 156-8506, Japan

Full list of author information is available at the end of the article
}

brains, namely, 6 tau isoforms in $\mathrm{AD}, 3 \mathrm{R}$ tau isoforms in Pick's disease, and 4R tau isoforms in PSP and CBD [1,2]. In addition, tau in PSP and tau in CBD are biochemically distinguished by the banding pattern of the C-terminal fragments [3]. However, it remains unclear why different tau isoforms accumulate in different diseases and how they lead to the formation of abnormal filamentous structures and pathologies.

Isoform-specific tau antibodies are useful tools for immunohistochemical and biochemical studies of tau species in diseased brains. In particular, RD3 and RD4 [4], which are specific antibodies to $3 R$ and $4 R$ tau isoforms, respectively, have been widely used to investigate tau pathologies [5-7]. One of the present authors (M.H.) had found that the asparagine residue at position 279 (N279), located in the RD4 epitope, was detected mostly as aspartic acid owing to deamidation of asparagine when PHF-tau in AD brains was subjected to protein sequencing and LC/MS/ 
MS analysis after digestion with lysyl endopeptidase [8]. Here, we show that the irreversible post-translational deamidation takes place at N279 (N279D) in the RD4 epitope of tau in AD, but not CBD or PSP, and this modification abrogates the immunoreactivity to RD4. We raised an antiserum against RD4 peptide with N279D in rabbit, and showed that it specifically recognizes $4 \mathrm{R}$ tau isoforms regardless of deamidation and strongly stained tau in $\mathrm{AD}$ brain. We further show that mutant tau with N279D substitution has a reduced ability to bind to microtubules and to promote their assembly. These results have important implications for immunohistochemical and other studies aimed at understanding the molecular mechanisms of tau accumulation in $\mathrm{AD}$ and other tauopathies.

\section{Results}

Low immunoreactivities of tau in $A D$ and tau deamidated at $\mathrm{N} 279$ to RD4

When Sarkosyl-insoluble fractions of tau from AD, PSP and CBD brains were analyzed by immunoblotting with T46 and RD4, we noticed a lower immunoreactivity of
RD4 with abnormal tau in AD compared to that in both PSP and CBD (Figure 1a,b). T46, a monoclonal antibody to the C-terminal region of tau, strongly labeled triplet bands of phosphorylated full-length tau in AD together with smearing substances, and doublet bands together with C-terminal fragments of tau in CBD and PSP (Figure 1a). In contrast, RD4 (1:1000 dilution) stained tau in CBD and PSP relatively strongly, but barely stained the tau bands, and especially the smears, in AD (Figure 1b), though both RD4 and T46 labeled Sarkosyl-soluble tau in these brains (Figure 1c,d). These results suggested that there might be some modification in the RD4 epitope or its vicinity on tau in $\mathrm{AD}$ abrogating immunoreactivity. The low affinity of RD4 for tau in AD is consistent with the original report [4], which noted that the RD4 titer appeared to be considerably weaker than those of TP70 and RD3. To confirm that the asparagine residue at position 279 (N279) on tau was deamidated in AD, we performed LC/MS/MS analyses of tryptic peptides of Sarkosyl-insoluble tau prepared from AD brains. As shown in Figure 1e, almost all the VQIINK peptide

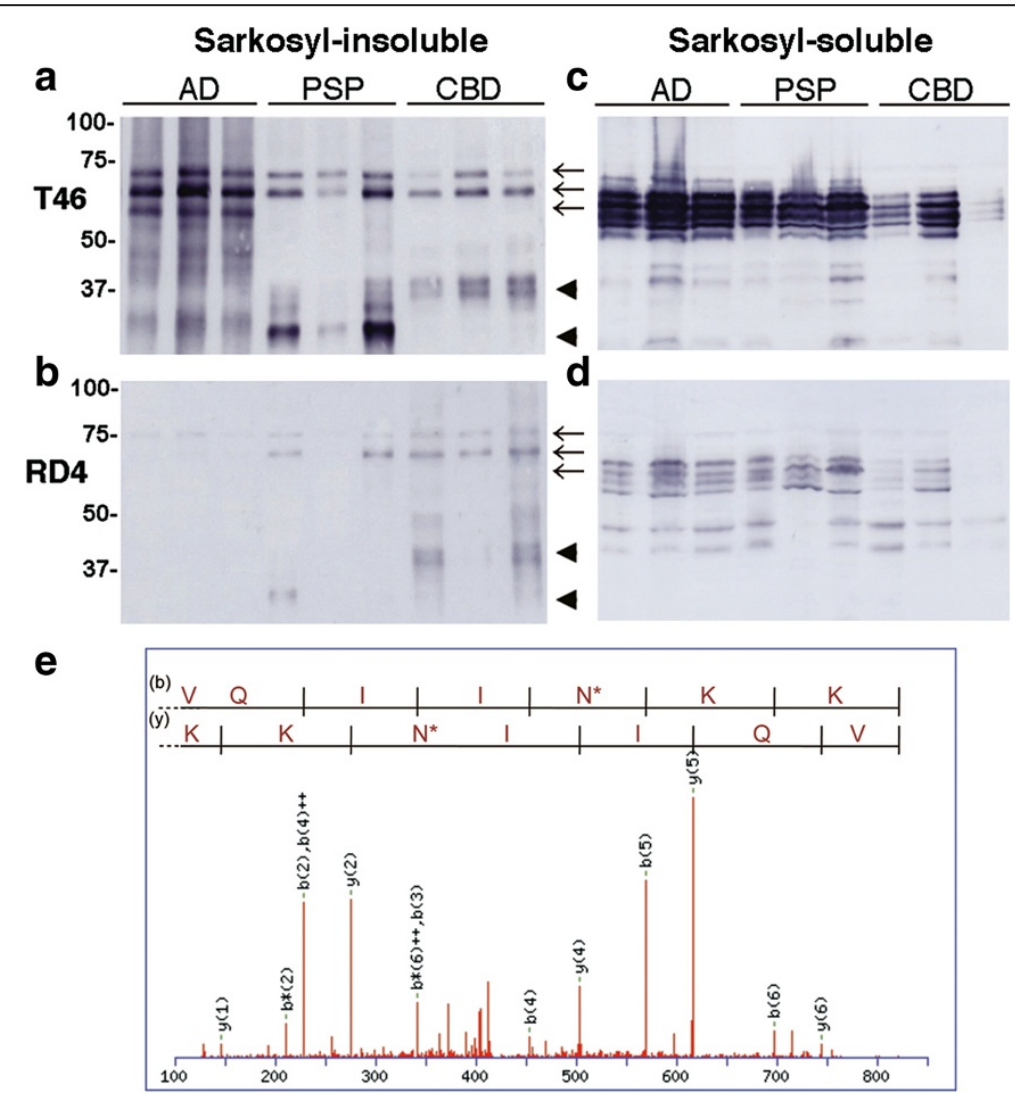

Figure 1 Immunoblot and LC/MS/MS analyses showed a lower immunoreactivity of RD4 with tau in AD and deamidation at N279. Immunoblot analysis of Sarkosyl-insoluble $(\mathbf{a}, \mathbf{b})$ and soluble $(\mathbf{c}, \mathbf{d})$ tau from AD, PSP and CBD brains (three cases for each disease) with anti-tau monoclonal antibodies T46 (a, c) and RD4 (b, d). Arrows indicated the positions of the 60, 64, 68 kDa triplet tau bands in AD brains, and arrowheads indicate the $\sim 33$ and $\sim 37 \mathrm{kDa}$ C-terminal fragments that distinguish PSP and CBD. Identification of deamidated amino acid residue by nano-electrospray tandem mass spectrometry (e). Product ion spectrum of a mass signal of tryptic peptide VQIINK detected in Sarkosyl-insoluble tau from AD brain, showing the $b$ and $y$ ion series. These results identify the site of deamidation as N279, indicated by $\mathrm{N}^{*}$. 
derived from $4 \mathrm{R}$ tau isoform was detected as VQIIN*K (* indicates deamidation) (Figure 1e), while Q276 was normal, strongly suggesting that N279 is extensively deamidated in tau in AD.

\section{RD4 cannot recognize 4R tau with deamidation at N279}

Since N279 is located in the RD4 epitope, we next examined the effect of deamidation of N279 on immunoreactivity to
RD4. Substitution of N279 to aspartic acid was introduced into $4 \mathrm{R} 1 \mathrm{~N}$ human tau isoform by site-directed mutagenesis, and immunoreactivity to mutant (N279D)4R tau before and after phosphorylation by protein kinase A was compared with that of wild-type tau. As shown in Figure 2(a-d), RD4 was not able to recognize N279D-4R tau regardless of the phosphorylation state of Ser262, which is located near the RD4 epitope. To further investigate the
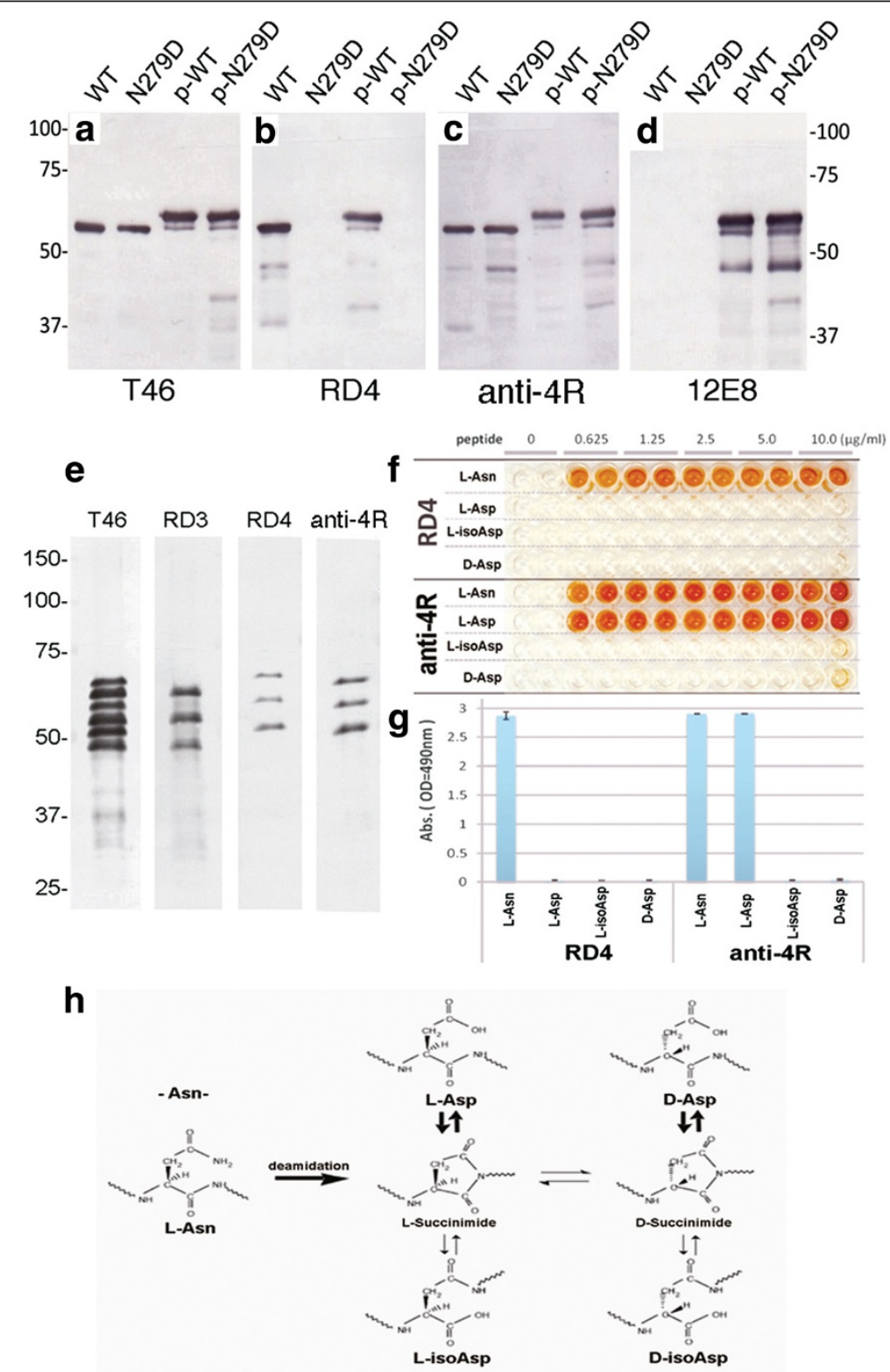

Figure 2 RD4 cannot recognize N279D-4R tau, but new anti-4R labels both WT and N279D-4R tau equally. Immunoblot analysis of wild-type (WT) and N279D mutant tau before (WT, N279D) and after (p-WT, p-N279D) phosphorylation with PKA, using T46 (a), RD4 (b), anti-4R (c) and 12E8 (d) antibodies. Immunoblot analysis of six recombinant human tau isoforms with T46, RD3, RD4 and anti-4R antibodies (e). Specificities of RD4 (1:1000 dilutions) and anti-4R (1:3000 dilutions) antibodies for synthetic peptides, L-Asn (wild-type), L-Asp, L-isoAsp and D-Asp peptides $(0.625 \sim 10 \mu \mathrm{g} / \mathrm{mL}$ ) tested by ELISA assay (f). Quantitation of the ELISA results (the mean of absorbance at $490 \mathrm{~nm}$ on $1.25 \mu \mathrm{g} / \mathrm{mL}$ peptide is shown (g). Pathways for deamidation of asparaginyl residues (h). L-Asn residue can be converted spontaneously via a succinimidyl intermediate to form L-Asp, D-Asp. L-isoAsp and D-isoAsp residue (modified from Ref. [9]). 
deamidation of $\mathrm{N} 279$ of tau, we immunized a rabbit with a synthetic peptide, VQIIDKKLDLSNVQSKC, which is the RD4 antigen peptide with substitution of N279 to Asp. The antiserum anti-4R labeled both wild-type (WT) and N279D-4R tau equally (Figure 2c) and the immunoreactivity was unaffected by phosphorylation of Ser262/356 with PKA (Figure 2c). The anti-4R antibody specifically bound with recombinant human $4 \mathrm{R}$ tau isoforms, as did RD4, but did not react with the $3 \mathrm{R}$ tau isoforms (Figure $2 \mathrm{e}$ ). The specificities of RD4 and anti-4R antibodies were further analyzed by means of ELISA assay using the antigen peptide of RD4 (L-Asn), the peptide with N279D substitution (LAsp), the peptide with L-isoAsp substitution (L-isoAsp) and the peptide with N279 D-Asp substitution (D-Asp) (Figure 2f,g). These modifications are known to be related to deamidation of Asn residue (Figure 2h). RD4 failed to react with L-Asp (antigen peptide with N279D substitution), whereas anti-4R reacted almost equally with L-Asn (wild-type) and L-Asp peptide. Neither RD4 nor anti-4R reacted with D-Asp or L-isoAsp peptide (Figure 2f,g).

\section{Antiserum against peptide with deamidation of N279 strongly stained tau smears in AD}

The immunoreactivity of anti-4R was compared to that of RD4, RD3 and T46 in immunoblotting of Sarkosylinsoluble tau from tauopathy brains (Figure 3). Samples of insoluble tau from three AD (lane 1-3), two PSP (lane 3,4) and two CBD (lane 6, 7) cases were examined (Figure 3a-d). RD4 faintly stained only two bands at 64 and $68 \mathrm{kDa}$ in $\mathrm{AD}$ brains, whereas it stained several tau fragments in PSP and CBD, in addition to the two bands at 64 and 68 (Figure $3 \mathrm{~b}$ ). In contrast, anti-4R stained tau bands and smears in AD, like RD3, and this staining was much stronger than that of tau bands and smears in PSP and CBD (Figure 3d). These results strongly suggest that tau in $\mathrm{AD}$ brains is predominantly deamidated at N279, and that the levels of deamidation are much lower in tau from PSP and CBD brains.

Next, we compared the immunostaining of tau pathologies with RD3, RD4 and anti-4R on formalin-fixed brain sections of AD (Figure 4). None of the three antibodies stained tau on formalin-fixed sections in the absence of formic acid treatment or autoclaving (not shown), suggesting that the epitopes of these antibodies are masked. After autoclaving or formic acid treatment, neurofibrillary tangles (NFTs) and neuropil threads (NTs) were detected with these antibodies, and dual treatment with both autoclaving and formic acid strongly enhanced the staining (not shown). The new anti-4R antibody stained intracellular NFTs and NTs more extensively than did RD4 (Figure 4). This was most evident in the CA1 region, where anti-4R strongly stained RD3+/RD4NFTs (Figure 4d,f). This result indicates that the RD4 epitope is deamidated in pathological tau from $\mathrm{AD}$ brain,

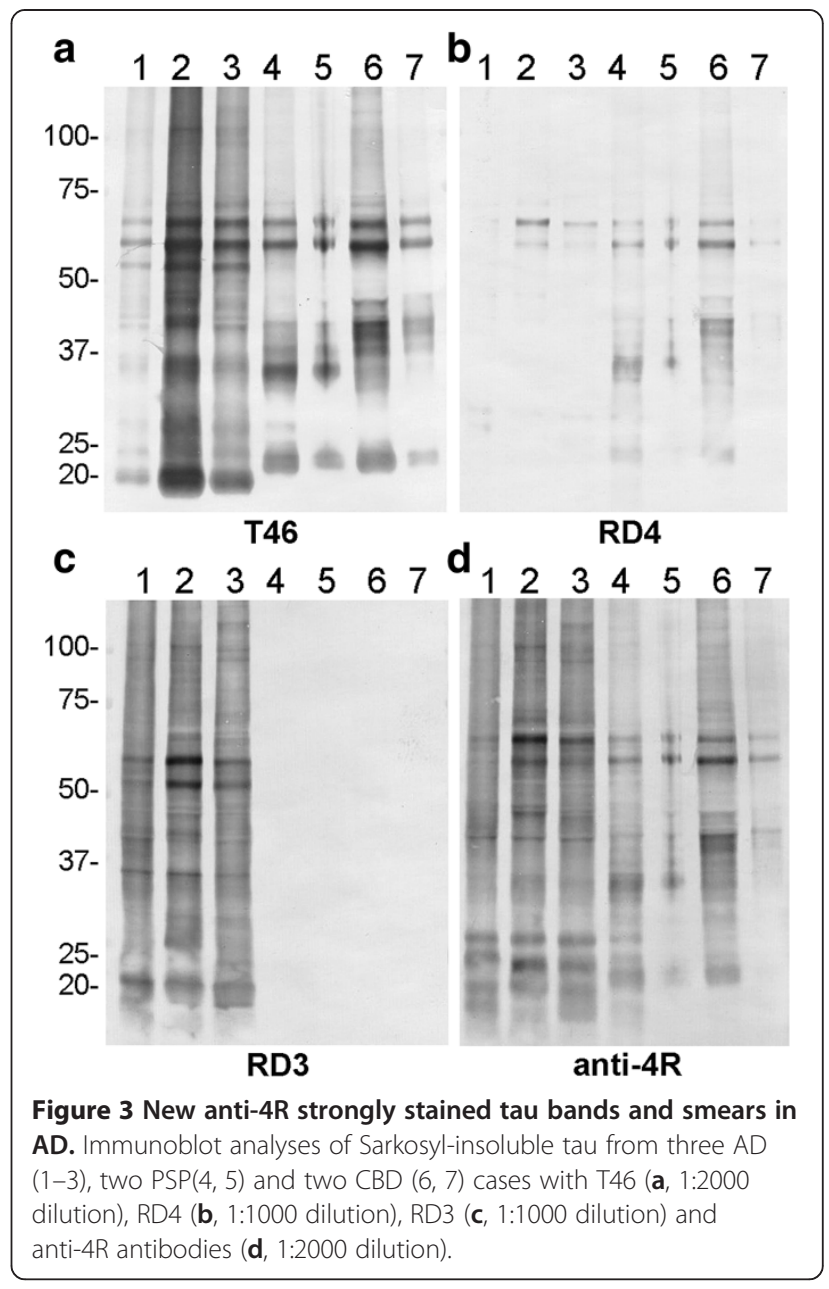

especially in RD3+/RD4- NFTs. RD3 stained abundant ghost tangles in entorhinal cortex and NFTs in CA1, but failed to stain fine processes of NFTs and NTs (Figure 4), as previously reported [10]. Anti-4R also failed to detect ghost tangles in entorhinal cortex.

\section{Deamidation of $\mathrm{N} 279$ reduces the ability of tau to bind microtubules}

N279 is located in one of the repeat regions of tau that are involved in binding to microtubules. Therefore, we tested whether the deamidation influences the role of tau in microtubule binding and assembly. First, the ability of N279D mutant tau to mediate polymerization of tubulin was compared to that of wild-type tau by monitoring the turbidity after mixing tubulin with tau. The N279D mutant tau showed a reduced ability to promote microtubule assembly (Figure 5a). We next investigated the binding ability of the N279D mutant tau to taxolstabilized microtubules. Tau bound and unbound to microtubules was separated by centrifugation and the levels were quantitated by means of SDS-PAGE and CBB staining (Figure 5b,c). When the amount of tau was 


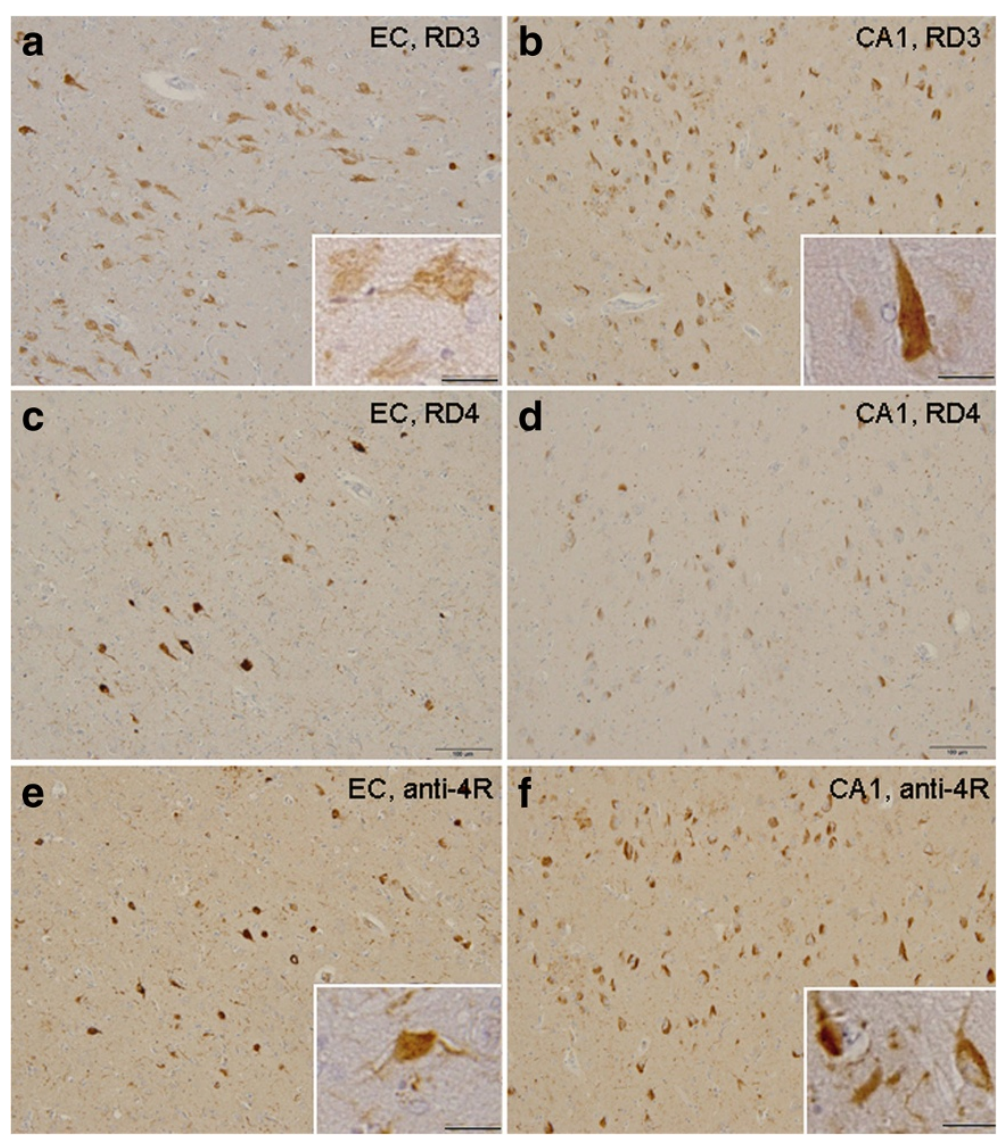

Figure 4 New anti-4R antibody stained intracellular NFTs more extensively than did RD4. Immunostaining of entorhinal cortex (EC) and CA1 sections of AD brain after autoclaving and formic acid treatments, using RD3 (a, b), RD4 (c, $\mathbf{d})$ and anti-4R (e, $\mathbf{f})$ antibodies. NFTs with higher magnification are shown in insets. Bar $=100 \mu \mathrm{m}$ ( $25 \mu \mathrm{m}$ in insets).

increased in the presence of a constant amount of microtubules, the binding affinity and the microtubule assembly-promoting activity of N279D mutant tau were both found to be much lower than those of WT tau, clearly indicating that deamidation of $\mathrm{N} 279$ reduced the functional activity of tau (Figure 5b,c). Since several positively charged residues have been shown to be important for the ability of tau to promote microtubule assembly, negative charge arising from deamidation of N279 may affect the interaction.

\section{Discussion}

Our present results indicate that the N279 on the RD4 epitope is extensively deamidated in pathological tau from AD brain. Because the widely used RD4 antibody is unreactive to the deamidated epitope, the level of $4 R$ tau isoforms in $\mathrm{AD}$ brain will have been markedly underestimated in previous immunohistochemical and biochemical analyses using RD4 antibody. Deamidation is an irreversible, non-enzymatic reaction, in which the amidecontaining side chain is removed from asparagine or glutamine. It is known to be a marker for aging in proteins with long life-spans, and, for example, many deamidation sites have been identified in crystallins [11], the major proteins of the eye lens. In biochemical deamidation, the side chain of an asparagine residue attacks the amide group, forming a succinimide intermediate, which, upon hydrolysis, affords either aspartate or isoaspartate [12]. Isoaspartate formation from asparagine residues of tau has been reported in AD brains $[9,13]$, but deamidation has been less well investigated. Nevertheless, deamidation is important because it alters the charge of the amino acid residue, and this can markedly affect protein structure and interaction with other proteins. Therefore, deamidation of N279 may have an effect on tau similar to that of missense mutations in FTDP-tau, many of which affect the ability of tau to promote microtubule assembly or to self-aggregate into amyloid fibrils. Indeed, substitution of N279 to Asp greatly reduced the ability of tau to promote microtubule assembly (Figure 5). However, we did not observe any accelerating effect on tau fibril formation (data not shown). Further studies are needed, but it is possible that the deamidation may be a consequence of aging of tau in paired helical filaments (PHF). 


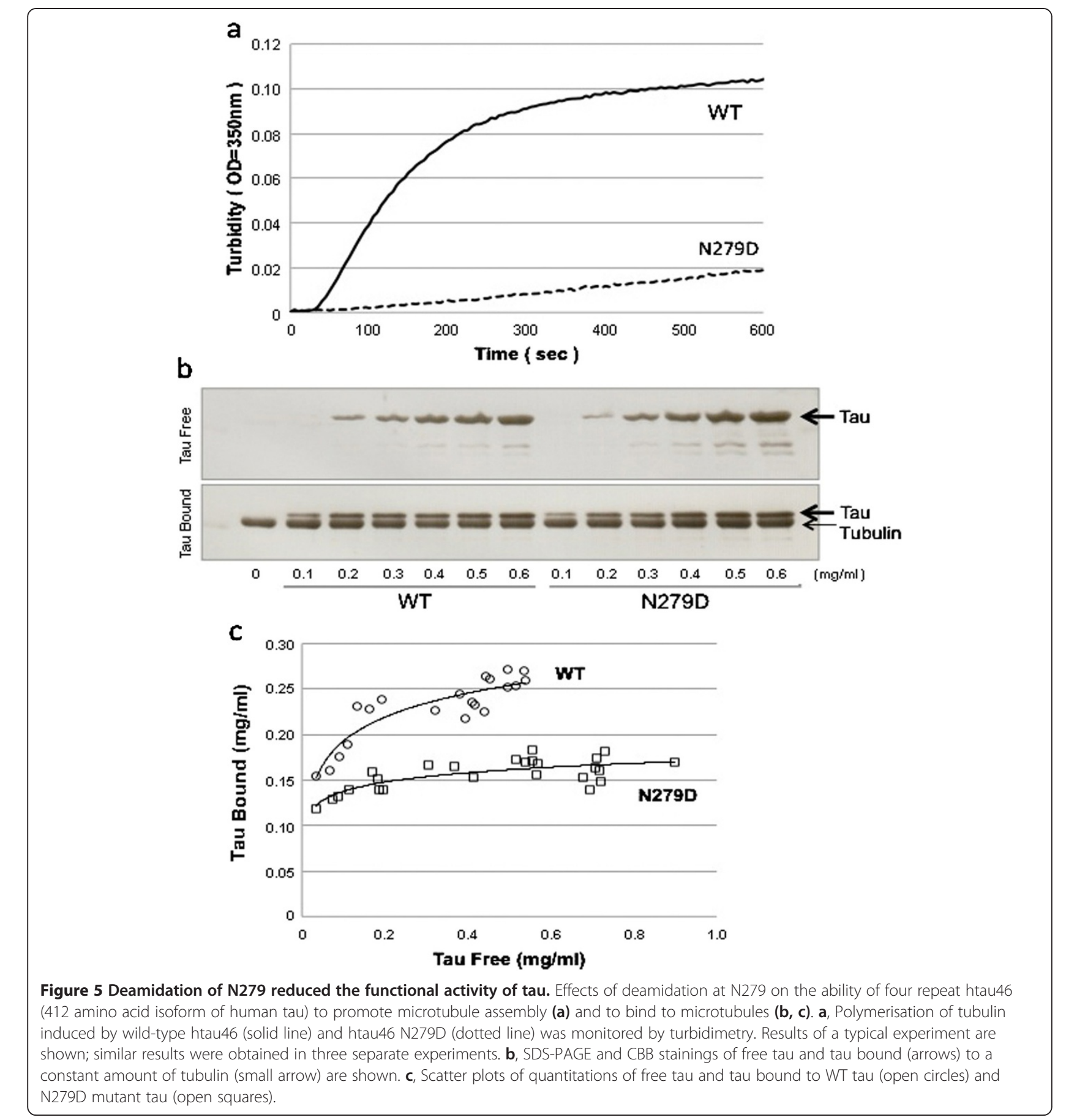

Other potential post-translational modifications in the RD4 epitope include acetylation and methylation on K280 [14-16]. Using antibodies specific for tau acetylated at lysine 280, significant acetylated-tau pathology has been found with a distribution pattern similar to that of hyperphosphorylated tau [15]. However, in our protein chemical analyses (including LC/MS/MS) of AD tau, such modification has not been clearly detected. It is possible that the modification is hardly detectable in $\mathrm{LC} /$
MS/MS. But it is also possible that antibodies to acetylated K280 peptide may recognize a tau epitope exposed as a result of conformational change. It remains to be investigated whether the acetylation or methylation alters immunoreactivity to RD4 or whether deamidation of N279 influences immunoreactivity to acetylated K280.

The results of this study have implications for the molecular mechanisms of tau assembly. The RD4 immunoreactivity of $\mathrm{AD}$ tau (composed of $3 \mathrm{R}$ and $4 \mathrm{R}$ tau) is 
different from that of CBD tau and PSP tau (composed of $4 \mathrm{R}$ tau), suggesting that the tau filament core structures may be different. Indeed, abnormal tau filaments characteristic of each disease have been described [17]. It seems reasonable to speculate that the RD4 epitope is integrated in the filament cores in CBD and PSP, making it resistant to deamidation and degradation. However, further analyses will be needed to understand the structures of tau in CBD, PSP and other tauopathies.

Prion-like spreading of intracellular pathological proteins or template (seed)-dependent conversion of normal protein to abnormal forms are candidate molecular mechanisms for involvement in the pathogenesis and progression of neurodegenerative diseases including $\mathrm{AD}$ [18-21]. The biochemical and structural differences of tau in $A D$ from that in $4 R$ tauopathies found in this study may therefore have implications for prion-like propagation of tau. Heterodimeric tau composed of both $3 \mathrm{R}$ tau and $4 \mathrm{R}$ tau with an amyloid-like conformation may act as a template for converting normal $3 R$ and $4 R$ tau to the abnormal structures seen in neurons, forming unique PHF structures composed of both $3 \mathrm{R}$ and $4 \mathrm{R}$ tau. Therefore, site-specific antibodies are important tools for immunohistochemical and biochemical studies of the role of tau in neurodegenerative diseases.

\section{Conclusions}

We conclude that extensive irreversible post-translational deamidation takes place at asparagine residue 279 (N279) in the RD4 epitope of tau in Alzheimer's disease (AD), but not corticobasal degeneration (CBD) or progressive supranuclear palsy (PSP), and this modification abrogates the immunoreactivity to RD4. An antiserum raised against deamidated RD4 peptide specifically recognized 4R tau isoforms, regardless of deamidation, and strongly stained tau in AD brain.

\section{Methods}

\section{Human brain tissues}

Human brain tissues were obtained from The Manchester Brain Bank, University of Manchester (Manchester, UK), Tokyo Metropolitan Institute of Gerontology (Tokyo, Japan) and NCNP Hospital (Tokyo, Japan). This study was approved by the local research ethics committees of Tokyo Institute of Psychiatry and Tokyo Metropolitan Institute of Medical Science. The subjects included three patients diagnosed with AD, three with PSP and three with CBD, neuropathologically confirmed by immunohistochemistry with antibodies to tau, A $\beta, \alpha$-synuclein and TDP-43.

\section{Preparation of sarkosyl-insoluble fractions}

Brain samples (0.5 g) from patients with AD, PSP and CBD were each homogenized in $10 \mathrm{ml}$ of homogenization buffer (HB: 10 mM Tris- $\mathrm{HCl}, \mathrm{pH} 7.5$ containing $0.8 \mathrm{M}$
$\mathrm{NaCl}, 1 \mathrm{mM}$ EGTA, $1 \mathrm{mM}$ dithiothreitol). Sarkosyl was added to the lysates (final concentration: $2 \%$ ), which were then incubated for $30 \mathrm{~min}$ at $37^{\circ} \mathrm{C}$ and centrifuged at $20,000 \mathrm{~g}$ for $10 \mathrm{~min}$ at $25^{\circ} \mathrm{C}$. The supernatant was divided into eight tubes (each $1.3 \mathrm{~mL}$ ) and centrifuged at $100,000 \mathrm{~g}$ for $20 \mathrm{~min}$ at $25^{\circ} \mathrm{C}$. The pellets were further washed with sterile saline $(0.5 \mathrm{~mL} /$ tube $)$ and centrifuged at 100,000 g for $20 \mathrm{~min}$. The resulting pellets were used as Sarkosyl-insoluble fraction (ppt).

\section{LC/MS/MS analysis of sarkosyl-insoluble tau}

Sarkosyl-insoluble tau from AD brains was subjected to SDS-PAGE using 4-20\% polyacrylamide gel (PAGE mini, Daiichi, Tokyo). After staining with Coomassie brilliant blue R-250 (CBB), the bands corresponding to the phosphorylated tau $(64$ and $68 \mathrm{kDa})$ were cut out. In-gel digestion of proteins with $1 \mu \mathrm{g} / \mathrm{ml}$ trypsin was carried out as described previously and the resulting peptides were analyzed by an ion-trap spectrometry (Velos Pro; Thermo Fisher Scientific Inc. Waltham, MA). The MS/MS data files were searched and analyzed using the Mascot Server (Matrix Science Inc., Boston, MA).

\section{Recombinant tau proteins}

Expression constructs for six human tau isoforms in plasmid pRK172 were kindly provided by Dr. Goedert. Sitedirected mutagenesis was used to change N279 to Asp (numbering refers to the 441-amino-acid isoform of human brain tau) in the four-repeat 412-amino-acid isoform (expressed from cDNA clone htau46). Wild-type and mutated tau proteins were expressed in Escherichia coli BL21(DE3) and purified as described previously[22]. For in vitro phosphorylation, purified tau $(10 \mu \mathrm{g} / \mathrm{ml})$ was incubated with PKA $(10,000 \mathrm{U} / \mathrm{ml}$; New England Biolabs, Beverly, MA) in $30 \mathrm{mM}$ Tris- $\mathrm{HCl}$ buffer ( $\mathrm{pH} 7.5)$ containing $0.1 \mathrm{mM}$ EGTA, $10 \mathrm{mM} \mathrm{MgSO} 4,0.8 \mathrm{mM}$ PMSF and $2 \mathrm{mM} \mathrm{ATP}$, at $30^{\circ} \mathrm{C}$ for $1 \mathrm{hr}$.

\section{Antibodies}

RD3 (directed to residues 209 224: Millipore), RD4 (residues 275 291: Millipore), T46 (residues 404 441: Invitrogen) and pS396 (phospho-Ser396: Calbiochem) were purchased. Antiserum anti-4R was raised against a synthetic peptide VQIIDKKLDLSNVQSKC which corresponds to residues 275 291 of human tau (441 residues), with substitution of N279 to Asp (Sigma Aldrich Japan). The peptide was conjugated to $\mathrm{m}$-maleimidobenzoyl- $\mathrm{N}$ hydrosuccinimide ester-activated keyhole limpet hemocyanin (KLH). The KLH-peptide complex (1 mg of each immunogen) emulsified in Freund's complete adjuvant was injected subcutaneously into a New Zealand White rabbit, followed by 5 weekly subcutaneous injections of $150 \mu \mathrm{g}$ KLH-peptide complex emulsified in Freund's 
incomplete adjuvant, starting 3 weeks after the first immunization.

\section{ELISA assay}

Each synthetic peptide consisting of residues 275 291 (VQIINKKLDLSNVQSKC) with the fifth position being replaced by L-Asp, L-isoAsp, or D-Asp was synthesized by the solid-phase method (Sigma Aldrich Japan) These peptides, L-Asn (wild-type), L-Asp, L-isoAsp, DAsp $(0.625 \sim 10 \mu \mathrm{g} / \mathrm{ml}$ in $50 \mathrm{mM}$ Tris- $\mathrm{HCl}, \mathrm{pH} 8.8)$ were coated onto microtitre plates (SUMILON) at $4{ }^{\circ} \mathrm{C}$ for $16 \mathrm{~h}$. The plates were blocked with $10 \%$ fetal bovine serum (FBS) in PBS, incubated with the first antibodies (RD4, 1:1000; anti-4R, 1:3000) diluted in 10\% FBS/PBS at room temperature for $1.5 \mathrm{~h}$, followed by incubation with HRP-goat anti-rabbit IgG (Bio-Rad) at 1:1000 dilution, and reacted with the substrate, $0.4 \mathrm{mg} / \mathrm{ml}$ o-phenylendiamine, in citrate buffer $(24 \mathrm{mM}$ citric acid, $51 \mathrm{mM} \mathrm{Na} \mathrm{HPO}_{4}$ ), The absorbance at $490 \mathrm{~nm}$ was measured using Plate Chameleon (HIDEX) as described [23].

\section{Microtubule assembly and tau binding}

Purified recombinant wild-type and mutant tau (htau46) proteins $(0.1 \mathrm{mg} / \mathrm{ml}, 2.3 \mu \mathrm{M})$ were incubated with bovine brain tubulin $(1 \mathrm{mg} / \mathrm{ml}, 20 \mu \mathrm{M}$, cytoskeleton) in assembly buffer at $37^{\circ} \mathrm{C}$, as described [22]. The assembly of tubulin was monitored in terms of the change in turbidity at $350 \mathrm{~nm}$. The binding assay was performed as described [24]. Briefly, purified tubulin was incubated at $37^{\circ} \mathrm{C}$ in the presence of $1 \mathrm{mM} \mathrm{GTP}$ and $20 \mu \mathrm{M}$ taxol. Tau protein was added at various concentrations and each mixture was incubated for $10 \mathrm{~min}$. The suspensions were centrifuged for $100,000 \mathrm{~g}$ at $37^{\circ} \mathrm{C}$. The resulting pellets were resuspended in $50 \mathrm{mM}$ PIPES pH 6.9, 1 mM EGTA, $0.2 \mathrm{mM} \mathrm{MgCl}_{2}, 5 \mathrm{mM}$ DTT, $0.5 \mathrm{M} \mathrm{NaCl}$. The pellets and supernatants (containing bound and free tau, respectively) were subjected to SDS-PAGE and stained with Coomassie brilliant blue R250. The gels were scanned at $400 \mathrm{dpi}$ on a gel scanner and evaluated using the software provided.

\section{Gel electrophoresis and immunoblotting}

Samples were run on gradient $4-20 \%$ or $10 \%$ polyacrylamide gels and electrophoretically transferred to PVDF membranes. Residual protein-binding sites were blocked by incubation with $3 \%$ gelatin (Wako) for $10 \mathrm{~min}$ at $37^{\circ} \mathrm{C}$, followed by overnight incubation at room temperature with the primary antibody. The membrane was then incubated for $1 \mathrm{hr}$ at room temperature with anti-rabbit IgG (BA-1000, Vector Lab) or anti-mouse IgG (BA2000 , Vector lab), then incubated for $30 \mathrm{~min}$ with avidin-horseradish peroxidase (Vector Lab), and the reaction product was visualized by using $0.1 \% 3,3$ - diaminobenzidine $(\mathrm{DAB})$ and $0.2 \mathrm{mg} / \mathrm{ml} \mathrm{NiCl}_{2}$ as the chromogen.

\section{Immunohistochemistry}

Formalin-fixed paraffin-embedded sections of AD brains were used for immunohistochemistry. The sections were pretreated by autoclaving for $10 \mathrm{~min}$ in $10 \mathrm{mM}$ sodium citrate buffer at $120^{\circ} \mathrm{C}$ and treated with $100 \%$ formic acid for $10 \mathrm{~min}$. Sections were washed with $10 \mathrm{mM}$ phosphatebuffered saline (PBS, pH 7.4) three times for $10 \mathrm{~min}$ each. Sections were blocked with 10\% normal serum and incubated overnight at room temperature with one of the primary antibodies in PBS. After washing, sections were incubated with biotinylated anti-mouse or rabbit secondary antibody for $2 \mathrm{~h}$, followed by biotinylated horseradish peroxidase complex (ABC, Vector) for $1 \mathrm{hr}$. The label was visualized with EnVision ${ }^{\mathrm{Tm}}(\mathrm{Dako})$. Sections were counterstained with hematoxylin.

\section{Competing interests}

The authors declare that they have no competing interests.

\section{Authors' contributions}

AD, MT, MS and TN performed biochemical and immunochemical studies. FK performed LC/MS/MS analysis. HK performed immunohistochemistry. TA helped for characterisation of antibody. HA, YS, HH, SM and DM performed neuropathological studies and analyses. MH performed study design, preparation of antibody, biochemical analyses and wrote the paper. All authors read and approved the final manuscript.

\section{Acknowledgements}

We acknowledge the support of Alzheimer's Research UK and Alzheimer's Society through their funding of the Manchester Brain Bank under the Brains for Dementia Research (BDR) initiative. This work was supported by a Grantin-Aid for Scientific Research (S) (JSPS KAKENHI 23228004 to M.H.), a Grant-inAid for Scientific Research (A) (JSPS KAKENHI 23240050 to M.H.), MHLW Grant (Number 12946221 to M.H.) and a Grant-in-Aid for Scientific Research on Innovative Area 'Brain Environment' (MEXT KAKENHI 24111556 to T.N).

\section{Author details}

'Department of Neuropathology and Cell Biology, Tokyo Metropolitan Institute of Medical Science, Setagaya-ku, Tokyo 156-8506, Japan. ${ }^{2}$ Histology center, Tokyo Metropolitan Institute of Medical Science, Setagaya-ku, Tokyo 156-8506, Japan. ${ }^{3}$ Dementia Research Project, Tokyo Metropolitan Institute of Medical Science, Setagaya-ku, Tokyo 156-8506, Japan. ${ }^{4}$ Department of Applied Biological Science, Faculty of Science and Technology, Tokyo University of Science, 2641 Yamazaki, Noda-shi, Chiba-ken 278-8510, Japan. ${ }^{5}$ Centre for Clinical and Cognitive Neuroscience, Institute of Brain Behavior and Mental Health, University of Manchester, Salford M6 8HD, UK.

${ }^{6}$ Department of Laboratory Medicine, National Center Hospital, NCNP, 4-1-1 Ogawahigashi, Kodaira, Tokyo 187-8502, Japan. ${ }^{7}$ Department of

Neuropathology, Tokyo Metropolitan Institute of Gerontology, Itabashi-ku, Tokyo 173-0015, Japan

Received: 17 August 2013 Accepted: 17 August 2013

Published: 21 August 2013

\section{References}

1. Umeda Y, Taniguchi S, Arima K, Piao YS, Takahashi H, Iwatsubo T, Mann D, Hasegawa M: Alterations in human tau transcripts correlate with those of neurofilament in sporadic tauopathies. Neurosci Lett 2004, 359:151-154.

2. Goedert M, Spillantini MG: Pathogenesis of the tauopathies. J Mol Neurosci 2011, 45:425-431.

3. Arai T, Ikeda K, Akiyama H, Nonaka T, Hasegawa M, Ishiguro K, Iritani S, Tsuchiya K, Iseki E, Yagishita S, Oda T, Mochizuki A: Identification of amino-terminally 
cleaved tau fragments that distinguish progressive supranuclear palsy from corticobasal degeneration. Ann Neurol 2004, 55:72-79.

4. de Silva R, Lashley T, Gibb G, Hanger D, Hope A, Reid A, Bandopadhyay R, Utton M, Strand C, Jowett T, Khan N, Anderton B, Wood N, Holton J, Revesz T, Lees A: Pathological inclusion bodies in tauopathies contain distinct complements of tau with three or four microtubule-binding repeat domains as demonstrated by new specific monoclonal antibodies. Neuropathol Appl Neurobiol 2003, 29:288-302.

5. de Silva R, Lashley T, Strand C, Shiarli AM, Shi J, Tian J, Bailey KL, Davies P, Bigio EH, Arima K, Iseki E, Murayama S, Kretzschmar H, Neumann M, Lippa C, Halliday G, Mackenzie J, Ravid R, Dickson D, Wszolek Z, Iwatsubo T, Pickering-Brown SM, Holton J, Lees A, Revesz T, Mann DM: An immunohistochemical study of cases of sporadic and inherited frontotemporal lobar degeneration using 3R-and 4R-specific tau monoclonal antibodies. Acta Neuropathol 2006, 111:329-340.

6. Togo T, Akiyama H, Iseki E, Uchikado H, Kondo H, Ikeda K, Tsuchiya K, de Silva R, Lees A, Kosaka K: Immunohistochemical study of tau accumulation in early stages of Alzheimer-type neurofibrillary lesions. Acta Neuropathol 2004, 107:504-508.

7. Piao YS, Tan CF, Iwanaga K, Kakita A, Takano H, Nishizawa M, Lashley T, Revesz T, Lees A, de Silva R, Tsujihata M, Takahashi H: Sporadic four-repeat tauopathy with frontotemporal degeneration, parkinsonism and motor neuron disease. Acta Neuropathol 2005, 110:600-609.

8. Hasegawa M, Morishima-Kawashima M, Takio K, Suzuki M, Titani K, Ihara Y: Protein sequence and mass spectrometric analyses of tau in the Alzheimer's disease brain. J Biol Chem 1992, 267:17047-17054.

9. Watanabe A, Takio K, Ihara Y: Deamidation and isoaspartate formation in smeared tau in paired helical filaments. Unusual properties of the microtubule-binding domain of tau. J Biol Chem 1999, 274:7368-7378.

10. Hara M, Hirokawa K, Kamei $\mathrm{S}$, Uchihara T: Isoform transition from fourrepeat to three-repeat tau underlies dendrosomatic and regional progression of neurofibrillary pathology. Acta Neuropathol 2013, 125:565-579.

11. Van Kleef FS, De Jong WW, Hoenders HJ: Stepwise degradations and deamidation of the eye lens protein alpha-crystallin in ageing. Nature 1975, 258:264-266.

12. Geiger T, Clarke S: Deamidation, isomerization, and racemization at asparaginyl and aspartyl residues in peptides. Succinimide-linked reactions that contribute to protein degradation. J Biol Chem 1987, 262:785-794.

13. Miyasaka T, Watanabe A, Saito Y, Murayama S, Mann DM, Yamazaki M, Ravid R, Morishima-Kawashima M, Nagashima K, Ihara Y: Visualization of newly deposited tau in neurofibrillary tangles and neuropil threads. J Neuropathol Exp Neurol 2005, 64:665-674.

14. Cohen TJ, Guo JL, Hurtado DE, Kwong LK, Mills IP, Trojanowski JQ, Lee VM: The acetylation of tau inhibits its function and promotes pathological tau aggregation. Nat Commun 2011, 2:252.

15. Irwin DJ, Cohen TJ, Grossman M, Arnold SE, Xie SX, Lee VM, Trojanowski JQ: Acetylated tau, a novel pathological signature in Alzheimer's disease and other tauopathies. Brain 2012, 135:807-818.

16. Min SW, Cho SH, Zhou Y, Schroeder S, Haroutunian V, Seeley WW, Huang EJ, Shen Y, Masliah E, Mukherjee C, Meyers D, Cole PA, Ott M, Gan L: Acetylation of tau inhibits its degradation and contributes to tauopathy. Neuron 2010, 67:953-966.

17. Arima K: Ultrastructural characteristics of tau filaments in tauopathies: immuno-electron microscopic demonstration of tau filaments in tauopathies. Neuropathology 2006, 26:475-483.

18. Clavaguera F, Akatsu H, Fraser G, Crowther RA, Frank S, Hench J, Probst A, Winkler DT, Reichwald J, Staufenbiel M, Ghetti B, Goedert M, Tolnay M: Brain homogenates from human tauopathies induce tau inclusions in mouse brain. Proc Natl Acad Sci U S A 2013, 110:9535-9540.

19. Masuda-Suzukake M, Nonaka T, Hosokawa M, Oikawa T, Arai T, Akiyama H, Mann DM, Hasegawa M: Prion-like spreading of pathological alpha-synuclein in brain. Brain 2013, 136:1128-1138

20. Hasegawa M, Nonaka T, Tsuji H, Tamaoka A, Yamashita M, Kametani F, Yoshida M, Arai T, Akiyama H: Molecular dissection of TDP-43 proteinopathies. J Mol Neurosci 2011, 45:480-485.

21. Nonaka T, Masuda-Suzukake M, Arai T, Hasegawa Y, Akatsu H, Obi T, Yoshida M, Murayama S, Mann DM, Akiyama H, Hasegawa M: Prion-like Properties of
Pathological TDP-43 Aggregates from Diseased Brains. Cell Rep 2013, 4:124-134.

22. Hasegawa M, Smith MJ, Goedert M: Tau proteins with FTDP-17 mutations have a reduced ability to promote microtubule assembly. FEBS Lett 1998, 437:207-210.

23. Masuda M, Hasegawa M, Nonaka T, Oikawa T, Yonetani M, Yamaguchi $Y$, Kato K, Hisanaga S, Goedert M: Inhibition of alpha-synuclein fibril assembly by small molecules: analysis using epitope-specific antibodies. FEBS Lett 2009, 583:787-791.

24. Gustke N, Steiner B, Mandelkow EM, Biernat J, Meyer HE, Goedert M, Mandelkow E: The Alzheimer-like phosphorylation of tau protein reduces microtubule binding and involves Ser-Pro and Thr-Pro motifs. FEBS Lett 1992, 307:199-205.

doi:10.1186/2051-5960-1-54

Cite this article as: Dan et al:: Extensive deamidation at asparagine residue 279 accounts for weak immunoreactivity of tau with RD4 antibody in Alzheimer's disease brain. Acta Neuropathologica Communications 2013 1:54

\section{Submit your next manuscript to BioMed Central and take full advantage of:}

- Convenient online submission

- Thorough peer review

- No space constraints or color figure charges

- Immediate publication on acceptance

- Inclusion in PubMed, CAS, Scopus and Google Scholar

- Research which is freely available for redistribution 